Essin Turhan, Talat Körpinar

\title{
BERTRAND MATE OF TIMELIKE BIHARMONIC LEGENDRE CURVES IN LORENTZIAN HEISENBERG GROUP $\mathrm{Heis}^{3}$
}

\begin{abstract}
In this paper, we study Bertrand mate of timelike biharmonic Legendre curves in the Lorentzian Heisenberg group $\mathrm{Heis}^{3}$. We characterize timelike biharmonic Legendre curves in terms of their curvature and torsion. Moreover, we obtain the position vectors of timelike biharmonic Legendre curves and we construct parametric equations of Bertrand mate of timelike biharmonic Legendre curves in the Lorentzian Heisenberg group $\mathrm{Heis}^{3}$.
\end{abstract}

\section{Introduction}

Bertrand curves are well-studied classical curves and may be defined by their property that any Bertrand curve shares its principal normals with another Bertrand curve, sometimes referred to as Bertrand mate [25]. Accordingly, Bertrand mates represent particular examples of offset curves [14] which are used in computer-aided design (CAD) and computer-aided manufacture (CAM). The distance between a Bertrand curve and its mate measured along the principal normal is known to be constant. This particular offset property may be used to show that any surface which is spanned by a one-parameter family of geodesic Bertrand curves of the same 'kind' admits a parallel surface of the same type. Moreover, application of the Wahlquist-Estabrook prolongation technique [4,24] to the underlying nonlinear Gauß-Mainardi-Codazzi equations reveals that these surfaces are integrable.

On the other hand, the theory of biharmonic functions is an old and rich subject. Biharmonic functions have been studied since 1862 by Maxwell and Airy to describe a mathematical model of elasticity. The theory of

1991 Mathematics Subject Classification: 31B30, 58E20.

Key words and phrases: Legendre curve, biharmonic curve, position vector, Heisenberg group. 
polyharmonic functions was developed later on, for example, by E. Almansi, T. Levi-Civita and M. Nicolescu.

Let $(M, g)$ and $(N, h)$ be Lorentzian manifolds and $\phi: M \longrightarrow N$ a smooth map. Denote by $\nabla^{\phi}$ the connection of the vector bundle $\phi^{*} T N$ induced from the Levi-Civita connection $\nabla^{h}$ of $(N, h)$. The second fundamental form $\nabla d \phi$ is defined by

$$
(\nabla d \phi)(X, Y)=\nabla_{X}^{\phi} d \phi(Y)-d \phi\left(\nabla_{X} Y\right), \quad X, Y \in \Gamma(T M) .
$$

Here $\nabla$ is the Levi-Civita connection of $(M, g)$. The tension field $\tau(\phi)$ is a section of $\phi^{*} T N$ defined by

$$
\tau(\phi)=\operatorname{tr} \nabla d \phi
$$

A smooth map $\phi$ is said to be harmonic if its tension field vanishes. It is well known that $\phi$ is harmonic if and only if $\phi$ is a critical point of the energy:

$$
E(\phi)=\frac{1}{2} \int h(d \phi, d \phi) d v_{g}
$$

over every compact region of $M$. Now let $\phi: M \longrightarrow N$ be a harmonic map. Then the Hessian $\mathcal{H}$ of $E$ is given by

$$
\mathcal{H}_{\phi}(V, W)=\int h\left(\mathcal{J}_{\phi}(V), W\right) d v_{g}, \quad V, W \in \Gamma\left(\phi^{*} T N\right) .
$$

Here the Jacobi operator $\mathcal{J}_{\phi}$ is defined by

$$
\begin{gathered}
\mathcal{J}_{\phi}(V):=\bar{\Delta}_{\phi} V-\mathcal{R}_{\phi}(V), \quad V \in \Gamma\left(\phi^{*} T N\right) \\
\bar{\Delta}_{\phi}:=\sum_{i=1}^{m}\left(\nabla_{e_{i}}^{\phi} \nabla_{e_{i}}^{\phi}-\nabla_{\nabla_{e_{i}} e_{i}}^{\phi}\right), \mathcal{R}_{\phi}(V)=\sum_{i=1}^{m} R^{N}\left(V, d \phi\left(e_{i}\right)\right) d \phi\left(e_{i}\right),
\end{gathered}
$$

where $R^{N}$ and $\left\{e_{i}\right\}$ are the Riemannian curvature of $N$, and a local orthonormal frame field of $M$, respectively.

Let $\phi:(M, g) \rightarrow(N, h)$ be a smooth map between two Lorentzian manifolds. The bienergy $E_{2}(\phi)$ of $\phi$ over compact domain $\Omega \subset M$ is defined by

$$
E_{2}(\phi)=\int_{\Omega} h(\tau(\phi), \tau(\phi)) d v_{g}
$$

A smooth map $\phi:(M, g) \rightarrow(N, h)$ is said to be biharmonic if it is a critical point of the $E_{2}(\phi)$.

The section $\tau_{2}(\phi)$ is called the bitension field of $\phi$ and the Euler-Lagrange equation of $E_{2}$ is

$$
\tau_{2}(\phi):=-\mathcal{J}_{\phi}(\tau(\phi))=0 .
$$

In [1] the authors completely classified the biharmonic submanifolds of the three-dimensional sphere, while in [2] there were given new methods to construct biharmonic submanifolds of codimension greater than one in the 
n-dimensional sphere. The biharmonic submanifolds into a space of nonconstant sectional curvature were also investigated. The proper biharmonic curves on Riemannian surfaces were studied in [3]. Inoguchi classified the biharmonic Legendre curves and the Hopf cylinders in three-dimensional Sasakian space forms [7]. Then, Sasahara gave in [19] the explicit representation of the proper biharmonic Legendre surfaces in five-dimensional Sasakian space forms.

The second variation formula for biharmonic maps in spheres was deduced [16] and the stability of certain classes of biharmonic maps in spheres was discussed in [12]. Also, in [20] there were given some sufficient conditions for the instability of Legendre proper biharmonic submanifolds in Sasakian space forms and the author proved the instability of Legendre curves and surfaces in Sasakian space forms.

Biharmonic functions are utilized in many physical situations, particularly in fluid dynamics and elasticity problems. Most important applications of the theory of functions of a complex variable were obtained in the plane theory of elasticity and in the approximate theory of plates subject to normal loading. That is, in cases when the solutions are biharmonic functions or functions associated with them. In linear elasticity, if the equations are formulated in terms of displacements for two-dimensional problems then the introduction of a stress function leads to a fourth-order equation of biharmonic type. For instance, the stress function is proved to be biharmonic for an elastically isotropic crystal undergoing phase transition, which follows spontaneous dilatation. Biharmonic functions arise when dealing with transverse displacements of plates and shells. They can describe the deflection of a thin plate subjected to uniform loading over its surface with fixed edges. Biharmonic functions also arise in fluid dynamics, particularly in Stokes flow problems (i.e., low-Reynolds-number flows). There are many applications for Stokes flow such as in engineering and biological transport phenomena (for details, see $[6,11]$ ). Fluid flow through a narrow pipe or channel, such as that used in micro-fluidics, involves low Reynolds number. See page flow through cracks and pulmonary alveolar blood flow can also be approximated by Stokes flow. Stokes flow also arises in flow through porous media, which have been long applied by civil engineers to groundwater movement. The industrial applications include the fabrication of microelectronic components, the effect of surface roughness on lubrication, the design of polymer dies and the development of peristaltic pumps for sensitive viscous materials. In natural systems, creeping flows are important in biomedical applications and studies of animal locomotion.

In this paper, we study Bertrand mate of timelike biharmonic Legendre curves in the Lorentzian Heisenberg group Heis ${ }^{3}$. We characterize time- 
like biharmonic Legendre curves in terms of their curvature and torsion. Moreover, we obtain the position vectors of timelike biharmonic Legendre curves and we construct parametric equations of Bertrand mate of timelike biharmonic Legendre curves in the Lorentzian Heisenberg group $\mathrm{Heis}^{3}$.

\section{Preliminaries}

The Heisenberg group $\mathrm{Heis}^{3}$ is a Lie group which is diffeomorphic to $\mathbb{R}^{3}$ and the group operation is defined as

$$
(x, y, z) *(\bar{x}, \bar{y}, \bar{z})=\left(x+\bar{x}, y+\bar{y}, z+\bar{z}-\frac{1}{2} \bar{x} y+\frac{1}{2} x \bar{y}\right) .
$$

The identity of the group is $(0,0,0)$ and the inverse of $(x, y, z)$ is given by $(-x,-y,-z)$. The left-invariant Lorentz metric on $\mathrm{Heis}^{3}$ is

$$
g=-d x^{2}+d y^{2}+(x d y+d z)^{2} .
$$

The following set of left-invariant vector fields forms an orthonormal basis for the corresponding Lie algebra:

$$
\left\{e_{1}=\frac{\partial}{\partial z}, e_{2}=\frac{\partial}{\partial y}-x \frac{\partial}{\partial z}, e_{3}=\frac{\partial}{\partial x}\right\}
$$

The characterising properties of this algebra are the following commutation relations:

$$
\left[e_{2}, e_{3}\right]=e_{1}, \quad\left[e_{3}, e_{1}\right]=0, \quad\left[e_{2}, e_{1}\right]=0
$$

with

$$
g\left(e_{1}, e_{1}\right)=g\left(e_{2}, e_{2}\right)=1, \quad g\left(e_{3}, e_{3}\right)=-1 .
$$

Proposition 2.1. For the covariant derivatives of the Levi-Civita connection of the left-invariant metric $g$, defined above the following is true:

$$
\nabla=\frac{1}{2}\left(\begin{array}{ccc}
0 & e_{3} & e_{2} \\
e_{3} & 0 & e_{1} \\
e_{2} & -e_{1} & 0
\end{array}\right),
$$

where the $(i, j)$-element in the table above equals $\nabla_{e_{i}} e_{j}$ for our basis

$$
\left\{e_{k}, k=1,2,3\right\}=\left\{e_{1}, e_{2}, e_{3}\right\} .
$$

We adopt the following notation and sign convention for Riemannian curvature operator:

$$
R(X, Y) Z=-\nabla_{X} \nabla_{Y} Z+\nabla_{Y} \nabla_{X} Z+\nabla_{[X, Y]} Z .
$$

The Riemannian curvature tensor is given by

$$
R(X, Y, Z, W)=g(R(X, Y) Z, W) .
$$


Moreover we put

$$
R_{i j k}=R\left(e_{i}, e_{j}\right) e_{k}, \quad R_{i j k l}=R\left(e_{i}, e_{j}, e_{k}, e_{l}\right),
$$

where the indices $i, j, k$ and $l$ take the values 1,2 and 3 .

$$
R_{121}=-\frac{1}{4} e_{2}, \quad R_{131}=-\frac{1}{4} e_{3}, \quad R_{232}=\frac{3}{4} e_{3},
$$

and

$$
R_{1212}=-\frac{1}{4}, \quad R_{1313}=\frac{1}{4}, \quad R_{2323}=-\frac{3}{4} .
$$

\section{Timelike biharmonic curves in the Lorentzian Heisenberg Group} $\mathrm{Heis}^{3}$

Let $\gamma: I \longrightarrow$ Heis $^{3}$ be a non geodesic timelike curve on the Lorentzian Heisenberg group $\mathrm{Heis}^{3}$ parametrized by arc length. Let $\{T, N, B\}$ be the Frenet frame fields tangent to the Lorentzian Heisenberg group $\mathrm{Heis}^{3}$ along $\gamma$ defined as follows:

$T$ is the unit vector field $\gamma^{\prime}$ tangent to $\gamma, N$ is the unit vector field in the direction of $\nabla_{T} T$ (normal to $\gamma$ ), and $B$ is chosen so that $\{T, N, B\}$ is a positively oriented orthonormal basis. Then, we have the following Frenet formulas:

$$
\begin{aligned}
\nabla_{T} T & =\kappa_{1} N \\
\nabla_{T} N & =\kappa_{1} T+\kappa_{2} B \\
\nabla_{T} B & =-\kappa_{2} N,
\end{aligned}
$$

where $\kappa_{1}=|\tau(\gamma)|=\left|\nabla_{T} T\right|$ is the curvature of $\gamma$ and $\kappa_{2}$ is its torsion and

$$
\begin{aligned}
& g(T, T)=-1, g(N, N)=1, g(B, B)=1, \\
& g(T, N)=g(T, B)=g(N, B)=0 .
\end{aligned}
$$

With respect to the orthonormal basis $\left\{e_{1}, e_{2}, e_{3}\right\}$ we can write

$$
\begin{aligned}
T & =T_{1} e_{1}+T_{2} e_{2}+T_{3} e_{3}, \\
N & =N_{1} e_{1}+N_{2} e_{2}+N_{3} e_{3}, \\
B & =T \times N=B_{1} e_{1}+B_{2} e_{2}+B_{3} e_{3} .
\end{aligned}
$$

Theorem 3.1. $\gamma: I \longrightarrow$ Heis $^{3}$ is a timelike biharmonic curve if and only if

$$
\begin{aligned}
\kappa_{1} & =\text { constant } \neq 0, \\
\kappa_{1}^{2}-\kappa_{2}^{2} & =1-B_{1}^{2}, \\
\kappa_{2}^{\prime} & =N_{1} B_{1} .
\end{aligned}
$$

Proof. Using Eq. (1.4) and Frenet formulas (3.1), we have Eq. (3.2). 
Theorem 3.2. Let $\gamma: I \longrightarrow$ Heis $^{3}$ be a timelike curve with constant curvature. If $\kappa_{2}^{\prime} \neq 0$, then $\gamma$ is not biharmonic.

Proof. We can use (2.3) to compute the covariant derivatives of the vector fields $T, N$ and $B$ as:

$$
\begin{aligned}
\nabla_{T} T= & T_{1}^{\prime} e_{1}+\left(T_{2}^{\prime}+T_{1} T_{3}\right) e_{2}+\left(T_{3}^{\prime}+T_{1} T_{2}\right) e_{3} \\
\nabla_{T} N= & \left(N_{1}^{\prime}+\frac{1}{2}\left(T_{2} N_{3}-T_{3} N_{2}\right)\right) e_{1}+\left(N_{2}^{\prime}+\frac{1}{2}\left(T_{1} N_{3}+T_{3} N_{1}\right)\right) e_{2} \\
& +\left(N_{3}^{\prime}+\frac{1}{2}\left(T_{2} N_{1}+T_{1} N_{2}\right)\right) e_{3} \\
\nabla_{T} B= & \left(B_{1}^{\prime}+\frac{1}{2}\left(T_{2} B_{3}-T_{3} B_{2}\right)\right) e_{1}+\left(B_{2}^{\prime}+\frac{1}{2}\left(T_{1} B_{3}+T_{3} B_{1}\right)\right) e_{2} \\
& +\left(B_{3}^{\prime}+\frac{1}{2}\left(T_{2} B_{1}+T_{1} B_{2}\right)\right) e_{3} .
\end{aligned}
$$

Using Frenet formulas (3.1) and Eq. (3.3), we have

$$
\begin{aligned}
T_{1}^{\prime} & =\kappa_{1} N_{1}, \\
N_{1}^{\prime}+\frac{1}{2}\left(T_{2} N_{3}-T_{3} N_{2}\right) & =\kappa_{1} T_{1}+\kappa_{2} B_{1}, \\
B_{1}^{\prime}+\frac{1}{2}\left(T_{2} B_{3}-T_{3} B_{2}\right) & =-\kappa_{2} N_{1} .
\end{aligned}
$$

Assume that $\gamma$ is biharmonic. Differentiating Eq. (3.2) with respect to $s$, we obtain

$$
\kappa_{2}^{\prime} \kappa_{2}=B_{1} B_{1}^{\prime}
$$

We substitute $\kappa_{2}^{\prime}=N_{1} B_{1}$ in Eq. (3.5), give

$$
\kappa_{2}=\frac{B_{1}^{\prime}}{N_{1}}
$$

Using Eq. (3.4) and Eq. (3.6), we get

$$
\kappa_{2}=\frac{1}{4}=\text { constant }
$$

Therefore also $\kappa_{2}$ is constant and we have a contradiction that is $\kappa_{2}^{\prime}=$ $N_{1} B_{1} \neq 0$.

Corollary 3.3. $\gamma: I \longrightarrow$ Heis $^{3}$ is timelike biharmonic if and only if

$$
\begin{aligned}
\kappa_{1} & =\text { constant } \neq 0, \\
\kappa_{2} & =\text { constant }, \\
N_{1} B_{1} & =0, \\
\kappa_{1}^{2}-\kappa_{2}^{2} & =1-B_{1}^{2} .
\end{aligned}
$$


Corollary 3.4. If $N_{1} \neq 0$, then $\gamma$ is not timelike biharmonic.

Proof. We use the third equation of Eq. (3.4), together with Theorem 3.1 complete the proof of the corollary.

Corollary 3.5. If $N_{1}=0$, then

$$
T(s)=\sinh \mu_{0} e_{1}+\cosh \mu_{0} \sinh \Omega(s) e_{2}+\cosh \mu_{0} \cosh \Omega(s) e_{3},
$$

where $\mu_{0} \in \mathbb{R}$.

\section{Bertrand mate of a timelike biharmonic Legendre curve in the Lorentzian HeisenberggGroup $\mathrm{Heis}^{3}$}

A curve $\gamma: I \longrightarrow H_{e i s}{ }^{3}$ is said to be Legendre if it is an integral curve of the contact distribution $\mathcal{D}=\operatorname{Ker} \omega$ equivalently, $\omega\left(\gamma^{\prime}\right)=0$.

Lemma 4.1. Let $\gamma: I \longrightarrow H_{e i s}^{3}$ is a timelike Legendre curve. Then,

$$
z^{\prime}(s)+x(s) y^{\prime}(s)=0 .
$$

Proof. Using the orthonormal left-invariant basis (2.1), we have

$$
\begin{aligned}
\gamma^{\prime}(s) & =x^{\prime}(s) \partial_{x}+y^{\prime}(s) \partial_{y}+z^{\prime}(s) \partial_{z} \\
& =x^{\prime}(s) e_{3}+y^{\prime}(s) e_{2}+\omega\left(\gamma^{\prime}(s)\right) \partial_{z} .
\end{aligned}
$$

Then, $\gamma(s)$ is a timelike Legendre curve iff

$$
\gamma^{\prime}(s)=x^{\prime}(s) e_{3}+y^{\prime}(s) e_{2}, \quad \omega\left(\gamma^{\prime}(s)\right)=z^{\prime}(s)+x(s) y^{\prime}(s) .
$$

We obtain Eq. (4.1) and lemma is proved.

LEMMA 4.2. If $\gamma(s)$ is a timelike Legendre curve, then

$$
x^{\prime}(s) e_{3}+y^{\prime}(s) e_{2}=x^{\prime}(s) \frac{\partial}{\partial x}+y^{\prime}(s) \frac{\partial}{\partial y}-x(s) y^{\prime}(s) \frac{\partial}{\partial z} .
$$

Theorem 4.3. Let $\gamma: I \longrightarrow$ Heis $^{3}$ is a timelike biharmonic Legendre curve. Then the position vector of the curve $\gamma=\gamma(s)$ is:

$$
\begin{aligned}
\gamma(s)= & \left(\frac{1}{\kappa_{1}} \sinh \left(\kappa_{1} s+\sigma\right)+c_{1}, \frac{1}{\kappa_{1}} \cosh \left(\kappa_{1} s+\sigma\right)+c_{2},\right. \\
& \left.\frac{s}{2 \kappa_{1}}-\frac{1}{4 \kappa_{1}} \sinh 2\left(\kappa_{1} s+\sigma\right)+\frac{c_{1}}{\kappa_{1}} \cosh \left(\kappa_{1} s+\sigma\right)+c_{3}\right),
\end{aligned}
$$

where $c_{1}, c_{2}, c_{3}, \sigma$ are constants of integration.

Proof. If $\gamma: I \longrightarrow \mathrm{Heis}^{3}$ is a timelike biharmonic Legendre curve, then we can write its position vector as follows:

$$
\gamma(s)=x(s) \partial_{x}+y(s) \partial_{y}+z(s) \partial_{z} .
$$


Differentiating Eq. (4.4) with respect to $s$ and by using the corresponding orthonormal left-invariant frame (2.1), we find

$$
\gamma^{\prime}(s)=x^{\prime}(s) e_{3}+y^{\prime}(s) e_{2}+\omega\left(\gamma^{\prime}(s)\right) \partial_{z} .
$$

Since $\left|\nabla_{T} T\right|=\kappa_{1}$, we obtain

$$
\Omega(s)=\left(\frac{\kappa_{1}-\sinh 2 \mu_{0}}{\cosh \mu_{0}}\right) s+\sigma,
$$

where $\rho \in \mathbb{R}$.

Using Eq. (3.8) and Lemma 4.1, we get

$$
\cosh \mu_{0}=1 \text { and } \sinh \mu_{0}=0 .
$$

Then from Eq. (4.4) and Eq. (4.5), we get

$$
\begin{aligned}
x^{\prime}(s) & =\cosh \left(\kappa_{1} s+\sigma\right), \\
y^{\prime}(s) & =\sinh \left(\kappa_{1} s+\sigma\right), \\
z^{\prime}(s)+x(s) y^{\prime}(s) & =\sinh \mu_{0}=0 .
\end{aligned}
$$

If the system Eq. (4.7) is integrated, we obtain

$$
\begin{aligned}
& x(s)=\frac{1}{\kappa_{1}} \sinh \left(\kappa_{1} s+\sigma\right)+c_{1}, \\
& y(s)=\frac{1}{\kappa_{1}} \cosh \left(\kappa_{1} s+\sigma\right)+c_{2}, \\
& z(s)=\frac{s}{2 \kappa_{1}}-\frac{1}{4 \kappa_{1}} \sinh 2\left(\kappa_{1} s+\sigma\right)+\frac{c_{1}}{\kappa_{1}} \cosh \left(\kappa_{1} s+\sigma\right)+c_{3} .
\end{aligned}
$$

Thus, the proof of Theorem 4.3 is completed.

The picture of $\gamma(s)$ at $c_{1}=c_{2}=c_{3}=0, \sigma=-1$ and $\kappa_{1}=1$ :

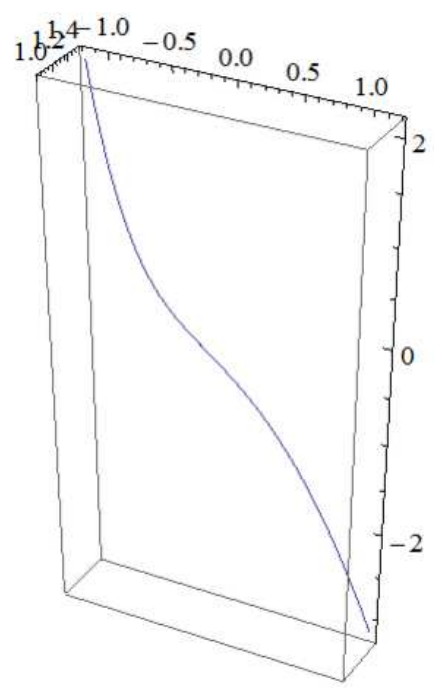


Definition 4.4. A curve $\gamma: I \longrightarrow$ Heis $^{3}$ with $\kappa_{1} \neq 0$ is called a Bertrand curve if there exists a curve $\widetilde{\gamma}: I \longrightarrow \mathrm{Heis}^{3}$ such that the principal normal lines of $\gamma$ and $\widetilde{\gamma}$ at $s \in I$ are equal. In this case $\widetilde{\gamma}$ is called a Bertrand mate of $\gamma[24]$.

Theorem 4.5. Let $\gamma: I \longrightarrow$ Heis $^{3}$ be a Bertrand curve parametrized by arc length. A Bertrand mate of $\gamma$ is as follows:

$$
\widetilde{\gamma}(s)=\gamma(s)+\lambda N(s), \quad \forall s \in I,
$$

where $\lambda$ is constant [24].

TheOREM 4.6. Let $\gamma: I \longrightarrow$ Heis $^{3}$ be a non-geodesic timelike horizontal biharmonic curve parametrized by arc length. If $\widetilde{\gamma}$ is a Bertrand mate of $\gamma$, then the parametric equations of $\widetilde{\gamma}$ are

$$
\begin{aligned}
\widetilde{x}(s) & =\left(\frac{1}{\kappa_{1}}+\lambda\right) \sinh \left(\kappa_{1} s+\sigma\right)+c_{1}, \\
(4.10) \widetilde{y}(s) & =\left(\frac{1}{\kappa_{1}}+\lambda\right) \cosh \left(\kappa_{1} s+\sigma\right)+c_{2}, \\
\widetilde{z}(s) & =-\left(\frac{1}{4 \kappa_{1}}+\frac{\lambda}{\kappa_{1}^{2}}\right) \sinh 2\left(\kappa_{1} s+\sigma\right)+\left(\frac{c_{1}}{\kappa_{1}}-\bar{c}_{2} \lambda\right) \cosh \left(\kappa_{1} s+\sigma\right) \\
& \frac{s}{2 \kappa_{1}}-\bar{c}_{1} \lambda s \cosh \left(\kappa_{1} s+\sigma\right)+c_{3},
\end{aligned}
$$

where $\bar{c}_{1}, \bar{c}_{2}, c_{1}, c_{2}, c_{3}, \sigma$ are constants of integration.

Proof. Using first equation of Eq. (3.3) and Eq. (4.6), we have

$$
\nabla_{T} T=\cosh \left(\kappa_{1} s+\sigma\right) e_{2}+\sinh \left(\kappa_{1} s+\sigma\right) e_{3} .
$$

By the use of Frenet formulas (3.1), we get

$$
N=\frac{1}{\kappa_{1}} \nabla_{T} T=\frac{1}{\kappa_{1}}\left(\cosh \left(\kappa_{1} s+\sigma\right) e_{2}+\sinh \left(\kappa_{1} s+\sigma\right) e_{3}\right) .
$$

From Eq. (2.1), we have

$$
\frac{\partial}{\partial x}=e_{3}, \quad \frac{\partial}{\partial y}=e_{2}+x e_{3}, \quad \frac{\partial}{\partial z}=e_{1} .
$$

Substituting Eq. (4.13) in Eq. (4.12), we have

$$
N=\left(\sinh \left(\kappa_{1} s+\sigma\right), \cosh \left(\kappa_{1} s+\sigma\right),-x(s) \cosh \left(\kappa_{1} s+\sigma\right)\right) .
$$

Next, we substitute Eq. (4.8) and Eq. (4.14) into Eq. (4.9), we get Eq. (4.10). 
The picture of $\widetilde{\gamma}(s)$ at $c_{1}=c_{2}=c_{3}=0, \sigma=-1$ and $\kappa_{1}=1$ :

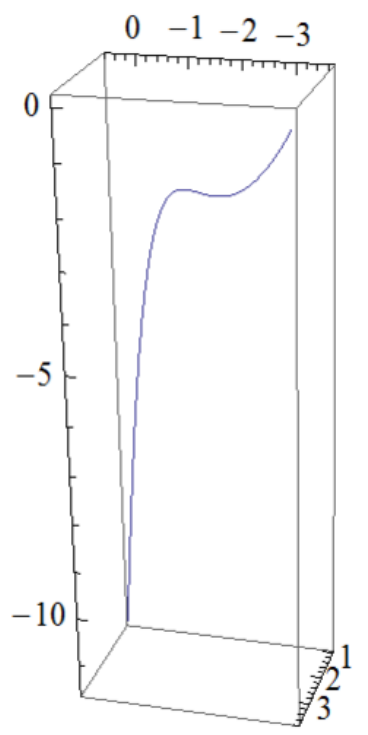

\section{References}

[1] R. Caddeo, S. Montaldo, C. Oniciuc, Biharmonic submanifolds of $\mathbb{S}^{3}$, Internat. J. Math. 12 (2001), 867-876.

[2] R. Caddeo, S. Montaldo, C. Oniciuc, Biharmonic submanifolds in spheres, Israel J. Math. 130 (2002), 109-123.

[3] R. Caddeo, S. Montaldo, P. Piu, Biharmonic curves on a surface, Rend. Mat. Appl. 21 (2001), 143-157.

[4] F. B. Estabrook, H. D. Wahlquist, Prolongation structures of nonlinear evolution equations II, J. Math. Phys. 17 (1976), 1293-1297.

[5] J. Eells, J. H. Sampson, Harmonic mappings of Riemannian manifolds, Amer. J. Math. 86 (1964), 109-160.

[6] J. Happel, H. Brenner, Low Reynolds Number Hydrodynamics with Special Applications to Particulate Media, Prentice-Hall, New Jersey, (1965).

[7] J. Inoguchi, Submanifolds with harmonic mean curvature in contact 3-manifolds, Colloq. Math. 100 (2004), 163-179.

[8] G. Y. Jiang, 2-harmonic isometric immersions between Riemannian manifolds, Chinese Ann. Math. Ser. A 7 (1986), 130-144.

[9] G. Y. Jiang, 2-harmonic maps and their first and second variation formulas, Chinese Ann. Math. Ser. A 7 (1986), 389-402.

[10] T. Körpınar, E. Turhan, On horizontal biharmonic curves in the Heisenberg group $\mathrm{Heis}^{3}$, Arab. J. Sci. Eng. Sect. A Sci., (in press).

[11] W. E. Langlois, Slow Viscous Flow, Macmillan, New York; Collier-Macmillan, London, (1964).

[12] E. Loubeau, C. Oniciuc, On the biharmonic and harmonic indices of the Hopf map, preprint, arXiv:math.DG/0402295 v1 (2004). 
[13] J. Milnor, Curvatures of left-invariant metrics on lie groups, Adv. Math. 21 (1976), 293-329.

[14] A. W. Nutbourne, R. R. Martin, Differential Geometry Applied to the Design of Curves and Surfaces, Ellis Horwood, Chichester, UK, 1988.

[15] B. O’Neill, Semi-Riemannian Geometry, Academic Press, New York (1983).

[16] C. Oniciuc, On the second variation formula for biharmonic maps to a sphere, Publ. Math. Debrecen 61 (2002), 613-622.

[17] Y. L. Ou, p-Harmonic morphisms, biharmonic morphisms, and nonharmonic biharmonic maps, J. Geom. Phys. 56 (2006), 358-374.

[18] S. Rahmani, Metriqus de Lorentz sur les groupes de Lie unimodulaires, de dimension trois, J. Geom. Phys. 9 (1992), 295-302.

[19] T. Sasahara, Legendre surfaces in Sasakian space forms whose mean curvature vectors are eigenvectors, Publ. Math. Debrecen 67 (2005), 285-303.

[20] T. Sasahara, Stability of biharmonic Legendre submanifolds in Sasakian space forms, preprint.

[21] E. Turhan, Completeness of Lorentz metric on 3-dimensional Heisenberg group, International Mathematical Forum 3 (13) (2008), 639-644.

[22] E. Turhan, T. Körpınar, Characterize on the Heisenberg Group with left invariant Lorentzian metric, Demonstratio Math. 42 (2) (2009), 423-428.

[23] E. Turhan, T. Körpınar, On characterization of timelike horizontal biharmonic curves in the Lorentzian Heisenberg group $\mathrm{Heis}^{3}$, Zeitschrift für Naturforschung A- A Journal of Physical Sciences, (in press).

[24] C. E. Weatherburn, Differential Geometry of Three Dimensions, Vol. I, Cambridge University Press, Cambridge, 1927.

[25] H. D. Wahlquist, F. B. Estabrook, Prolongation structures of nonlinear evolution equations, J. Math. Phys. 16 (1975), 1-7.

Essin Turhan and Talat Körpinar

FiRAT UNIVERSITY

DEPARTMENT OF MATHEMATICS

23119, ELAZIĞ, TURKEY

E-mail: essin.turhan@gmail.com

Received February 11, 2010; revised version July 6, 2010. 